\section{Medicina de urgencia y unidades de cuidados intensivos. Una alianza necesaria en busca de la mejoría de la atención de pacientes críticos}

\author{
BÁRBARA A. LARA ${ }^{1, \mathrm{a}}$, ALEJANDRO CATALDO ${ }^{2, \mathrm{~b}}$, RICARDO CASTRO $^{3, \mathrm{a}}$, \\ PABLO R. AGUILERA ${ }^{1}$, CAROLINA RUIZ ${ }^{3}$, MAX ANDRESEN ${ }^{3, c}$
}

\section{The impact of a better coordination between emergency and intensive care units in the care of critically ill patients}

The need for critical care services is increasing in Chile. Critical care beds and specialists in this area are scarce. In this article we discuss some aspects that hamper the care of critically ill patients from their arrival to the emergency department to their transfer to the ICU. Special emphasis is given to system saturation and its multiple causes. The benefits of an integrative approach between emergency medicine and critical care specialists are highlighted and some solutions are proposed to strengthen this partnership.

(Rev Med Chile 2016; 144: 917-924)

Key words: Critical Care; Emergency Medicine; Emergency Service, Hospital; Hospital Organization and administration.
'Sección de Medicina de

Urgencia, Facultad de Medicina,

Pontificia Universidad Católica de

Chile. Santiago, Chile.

2Departamento de Ingeniería

Industrial y de Sistemas Facultad

de Ingeniería, Pontificia

Universidad Católica de Chile.

Santiago, Chile.

${ }^{3}$ Departamento de Medicina

Intensiva, Facultad de Medicina

Pontificia Universidad Católica de

Chile. Santiago, Chile.

${ }^{\mathrm{a}} \mathrm{MPH}$.

bPhD.

${ }^{C} M S C$. FACP.

Recibido el 15 de septiembre de 2015, aceptado el 2 de junio de 2016.

Correspondencia a:

Dr. Max Andresen MsC, FACP

Departamento de Medicina

Intensiva, Facultad de Medicina,

Pontificia Universidad Católica

de Chile.

Marcoleta 367, Santiago Chile.

Teléfono: +56-2-29449169

andresen@med.puc.cl
L a medicina de urgencia (MDU) y los cuidados intensivos (CI) constituyen los eslabones centrales de la cadena de tratamiento de los pacientes críticos. La atención que reciben estos pacientes desde el pre-hospitalario hasta que son atendidos en la unidad de paciente crítico influye de manera importante en la evolución y el desenlace de aquellos pacientes de mayor gravedad ${ }^{1-6}$. La continuidad en la atención brindada requiere de colaboración estrecha entre ambas disciplinas (MDU y CI) para facilitar el manejo de éstos y optimizar los resultados en la condición de salud de los pacientes ${ }^{7}$. La medicina de urgencia y la medicina intensiva comparten una aproximación fisiopatológica y sistémica al enfrentamiento de pacientes, traspasando el clásico esquema de compartimientos, que históricamente ha dividido a las especialidades médicas. Una alianza de colaboración entre estas disciplinas podría aportar a contribuir a un manejo más adecuado de los pacientes críticos en nuestro país, ya que actualmente es común ver que los servicios de urgencia se encuentran sobrepasados y las camas críticas escasean. Es por esto que en este artículo discutimos los principales problemas que se enfrentan durante el flujo continuo de atención del paciente crítico y se plantean algunas oportunidades de mejora desde el punto de vista de procesos y estructura médico-organizacional.

\section{Evaluación inicial y destino de pacientes críticos}

El paciente crítico es definido por la Sociedad Americana de Medicina Intensiva, como aquel que se encuentra fisiológicamente inestable, que requiere soporte vital avanzado y una evaluación clínica estrecha con ajustes continuos de terapia según evolución ${ }^{8}$. La unidad de cuidados críticos 
(UCI) es sin duda el lugar que está dotado del personal humano y la infraestructura adecuada para cumplir con los requerimientos de cuidado anteriormente descritos. El análisis de la forma en la que funciona la Red de Atención de Salud, muestra que muchos pacientes críticamente enfermos son evaluados y manejados inicialmente en unidades fuera de la UCI, desde el mundo pre-hospitalario, servicios de urgencia (SU) y recuperaciones quirúrgicas?.

El advenimiento de protocolos que estandarizan terapias en pacientes críticos y la generación de unidades de accidente vascular cerebral o de dolor torácico fuera de la UCI, han mejorado la atención $\mathrm{n}^{10-13}$. Sin embargo, los recursos técnicos y humanos disponibles en estas unidades externas a las UCIs son insuficientes para manejar pacientes críticos más allá de su estabilización inicial. Además, y como consecuencia de la carencia de camas críticas, muchos pacientes deben permanecer fuera de las UCIs por tiempos prolongados.

Sumado al diagnóstico antes descrito, gran parte de los servicios de urgencia son aún manejados por médicos generales o especialistas con poca formación en urgencia y, por consiguiente, sin mayor preparación para el manejo inicial y menos definitivo de los pacientes más críticos. A pesar de que la especialidad de Medicina de Urgencia ha sido reconocida y existen programas de formación, aún faltan recursos que permitan acortar la brecha de especialistas que puedan hacerse cargo de estos pacientes en el mediano plazo en estos servicios.

\section{Saturación de los servicios de urgencia}

La saturación del servicio de urgencia es un fenómeno definido por un excesivo número de consultas al SU respecto a la capacidad real y efectiva que tiene éste para atenderlas de manera apropiada y oportuna ${ }^{8}$. A nivel mundial, la consolidación de la Medicina de Urgencia como especialidad ha coincidido con un aumento de las consultas a los $\mathrm{SU}^{14}$. La saturación y sobredemanda de estos servicios se ha vuelto un problema importante en la gestión de la salud pública. Un estudio multinacional, publicado el año 2011, describió la incidencia y las principales características del problema, destacando un aumento del número de visitas a una tasa de $5 \%$ por año e identificando la saturación como un problema mayor ${ }^{14}$. Los países que reportaron las tasas más bajas de saturación coincidían en tener un sistema sólido de atención primaria. Aunque sin duda existen muchos factores que determinan la saturación de los servicios de urgencia. Sí, el exceso de pacientes hospitalizados en la urgencia, por carencia de camas en el hospital, destaca como el principal determinante del problema. Esto último puede apreciarse en un estudio del año 2002, que reportó que 90\% de los SU de hospitales importantes de Estados Unidos de Norteamérica se veían diariamente sobrepasados por el número de consultas ${ }^{15}$.

Si bien en Chile no existen estadísticas específicas relacionadas con la saturación de los SU, de acuerdo al Departamento de Estadísticas e Información de Salud del Gobierno de Chile, las consultas a los SU y servicios de atención primaria de urgencia (SAPU) han aumentado significativamente los últimos años ${ }^{16}$. Por lo anterior, no parece equivocado suponer que nuestro escenario es similar al que enfrenta el resto del mundo.

Todo problema de saturación de un sistema tiene una causalidad multifactorial, y el SU no es la excepción. Aunque pudiese parecer ilógico, los principales determinantes de la saturación del $\mathrm{SU}$ parecen tener poca relación con los tiempos de ingreso (input), el tiempo de la evaluación del paciente y el tiempo que transcurre dentro del SU (este último ligado al rendimiento del sistema o throughput). Más bien la saturación tiene relación con situaciones que se originan más allá del SU (output). A pesar de que el número de visitas a los SU ha aumentado significativamente, este factor no pareciera ser el principal problema. En Estados Unidos de Norteamérica se pensaba que la ley EMTALA (Emergency Medical Treatment And Labor Act) era la principal causa de la saturación del sistema al obligar a todos los SU a evaluar y estabilizar todo paciente que requiera manejo urgente, independiente de su capacidad de pago. Sin embargo, múltiples estudios han demostrado que EMTALA sólo ha contribuido al aumento del número de visitas totales y no a la saturación del sistema ${ }^{17}$.

En Chile, la Ley de Urgencia establece que la atención médica ante un evento médico que requiere una intervención inmediata e impostergable (urgencia vital) o que deje secuelas importantes de no ser atendida de manera urgente, debe ser otorgada sin exigir respaldo financiero que 
condicione la atención en cualquier institución nacional, esto, sin importar la previsión de salud del paciente. Se podría extrapolar que, tal como en los Estados Unidos de Norteamérica, la Ley de Urgencia en Chile tampoco ha contribuido a este fenómeno de forma significativa, sin embargo, no hay estudios publicados que hayan evaluado su impacto. Por mucho tiempo también se pensó que el número de visitas de baja complejidad, catalogadas como "innecesarias", aportaban significativamente a la saturación del sistema. Así, se promovían intervenciones para enseñar a la población a evitar este tipo de consultas al SU y fomentar su acercamiento a servicios de menor complejidad. Sin embargo, un estudio de 1980 ha logrado desmitificar esta creencia. En este estudio se les preguntaba a los pacientes por la urgencia de su visita, y se concluyó que $25 \%$ de los pacientes identificados por el equipo médico por tener una patología de urgencia, pensaban que su consulta era de baja complejidad ${ }^{18}$. Un estudio de similares características también reportó un porcentaje de desacuerdo en cuanto a la valoración de la urgencia de las consultas entre médicos y pacientes ${ }^{19}$. Es así como la mayoría de los pacientes que consultan al SU lo hacen pensando que el síntoma que los aqueja es grave y que requieren resolución urgente. Entonces, si todos los motivos de consulta en SU podrían eventualmente ser síntoma de una patología que requiere manejo urgente, es responsabilidad del equipo médico y no del paciente evaluar la condición del paciente, y definir en base a esto, cuán oportuna o adecuada fue la consulta. Es más, estudios recientes han demostrado que las consultas “innecesarias" al SU no deberían interferir en lo absoluto con el cuidado y flujos de pacientes críticos en el SU².

Ahora bien, descartando que la llegada de pacientes y el proceso de atención de estos sea el principal determinante de la saturación de los SUs, centramos la mirada en el proceso de "espera de cama definitiva en el hospital", o "boarding". Este es uno de los factores que repetidamente ha sido identificado como el principal causante de saturación de los sistemas de urgencias. De ser así, se haría evidente que la principal causal de la saturación no está en el SU propiamente tal, sino en el sistema hospitalario completo, que es incapaz de acomodar adecuadamente los requerimientos de ingreso.

\section{Impacto de la saturación de los Servicios de Urgencia en los resultados finales de los pacientes críticos}

Se ha determinado que la saturación de los SUs aumenta el número de errores médicos. De acuerdo a la Joint Commission, 50\% de los eventos que causan complicaciones graves o muerte ocurren en el SU y un tercio de ellos ocurren en el contexto de saturación ${ }^{17}$. Muchos de estos errores son producidos por omisión de tratamiento, debido a la alta demanda que tiene el personal de salud, con un SU sobrepasado.

Cuando no se logra disponer oportunamente a los pacientes en las unidades que les corresponde según su complejidad y patología, se disminuye el tiempo en que el equipo tratante puede hacerse cargo de éste, disminuyendo la continuidad, óptimo seguimiento y tratamiento médico oportuno. Todo esto finalmente se traduce en aumento de la mortalidad ${ }^{21}$.

Por todas estas razones es que los pacientes críticos esperan más tiempo del establecido como tolerable antes de ser atendidos. El CDC (Centers for Disease Control and Prevention) reportó que $10 \%$ de los pacientes con categorización de urgente esperaban más de una hora por atención. Este problema es relevante, dado que muchas de estas patologías son tiempo-dependiente e intervenciones precoces llevan a mejores resultados. Además, como ya se dijo, muchos pacientes críticos atendidos en el servicio de urgencia esperan un período de tiempo prolongado en el SU antes de ser trasladados a la unidad de cuidado intensivo ${ }^{6}$.

A diferencia del servicio de urgencia, las UCIs cuentan con personal y recursos necesarios para manejar situaciones que requieren de monitoreo y manejo estrecho de pacientes críticos. Así, aquellos pacientes críticos que permanecen en el SU, donde el personal y los recursos se ven sobrepasados, difícilmente reciben un manejo óptimo. Esto queda justificado con diversos estudios publicados:

1. Un estudio de más de 50.000 pacientes mostró que pacientes críticos que permanecían en el $\mathrm{SU}$ por más de $6 \mathrm{~h}$ tienen mayores tasas de mortalidad durante su estadía en UCI $(10,7 \%$ vs $8,4 \%, p<0,01)$, mayor mortalidad hospitalaria $(17,4 \%$ vs $12,9 \%, \mathrm{p}<0,001)$ y una mediana de estadía hospitalaria más prolongada $(7,0 \text { días vs } 6,0 \text { días, } \mathrm{p}<0,001)^{22}$. 
2. Otro trabajo mostró que pacientes que requerían ventilación mecánica y eran trasferidos rápidamente a la UCI (menos de $2 \mathrm{~h}$ ) requirieron ventilación mecánica por menos días $(28,4$ vs $67,9 \mathrm{p}=0,02)$ y tendían a tener estadías hospitalaria más cortas ( 6,8 días vs 8,9 días $\mathrm{p}=0,0609)^{3}$.

3. Cardoso et al estudiaron 401 pacientes en UCI y la relación entre mortalidad y retraso en el traslado. La mortalidad del grupo de pacientes trasladados entre las 2 y $12 \mathrm{~h}$ desde su llegada a urgencia tuvieron una mortalidad de $43,4 \%$ versus $46,2 \%$ si la demora era de entre 12 a $18 \mathrm{~h}$ y de $52 \%$ con entre 18 a $24 \mathrm{~h}$ de retraso. Los autores estimaron que, por cada hora de atraso de traslado a UCI, la mortalidad aumentaba $1,5 \%{ }^{2}$.

Finalmente, según un estudio de cohorte con 619 pacientes con insuficiencia respiratoria o renal agudas hospitalizados en UCI dentro de las primeras $24 \mathrm{~h}$ de su ingreso al SU encontró que existe un aumento en el RR de $6 \%$ por cada hora de demora en traslado ${ }^{4}$.

En respuesta a todo lo expuesto anteriormente, en algunos países donde la medicina de urgencia ya está desarrollada, han literalmente llevado la UCI a la Urgencia, brindado "Cuidados Críticos de Urgencia" con los más altos estándares requeridos, hasta el momento en que los pacientes puedan ser finalmente dispuestos en las unidades manejadas por los especialistas en cuidados críticos $^{30}$.

\section{Situación nacional}

El Ministerio de Salud de Chile mantiene un registro de todos los traslados de pacientes desde los SUs de hospitales públicos a otros establecimientos, tanto privados como públicos. En la Tabla 1 se entrega un resumen por año, entre mayo del año 2009 y junio del año 2013. Según esta información, más de 70\% (7.392 de 10.397) de estos traslados han sido realizados desde el sistema público al privado, reflejando que el sistema público no posee la capacidad para resolver todos los requerimientos que llegan a sus servicios de urgencia y que requieren un número mayor de camas críticas.

Se estima que Chile cuenta con 1.334 camas críticas de adultos en hospitales públicos y su tiempo de estadía promedio es de 8,87 días. Con estos estos datos podemos estimar la capacidad de camas críticas del servicio público. Empleando la ecuación de Little (ecuación utilizada en Teoría de Colas para describir sistemas de espera), donde L es el número promedio de personas en el sistema (1.334 pacientes) y $\mathrm{W}$ es el tiempo de estadía promedio ( 8,87 días), se obtiene que el sistema tuvo capacidad para atender a 219.730 pacientes en 4 años (entre mayo de 2009 y junio de 2013).

Con esta información se puede inferir que de este total de requerimientos de UCI que llegan a los hospitales públicos de Chile, 95,42\% es atendido en el mismo establecimiento en que se presentó la urgencia, $1,32 \%$ es derivado a otro establecimiento público y $3,25 \%$ es derivado a un establecimiento privado. Los antecedentes indicados hablan sobre la falta de capacidad que posee el sistema público para hacerse cargo del número de consultas que se reciben en los SU, considerando solamente las consultas que requieren tratamiento en UCIs.

Si se realiza un análisis similar para la Región de Valparaíso, se observa que esta región cuenta con 148 camas críticas. Asumiendo un tiempo de estadía promedio de 8,61 días, se obtiene que el sistema de salud de esta región tuvo capacidad para atender a 25.123 pacientes (mayo de 2009 a junio de 2013), que se pueden asumir como egresos de pacientes desde las UCIs públicas de la Quinta Región durante esos 4 años. Con esta información, descrita en la Tabla 2, se puede inferir que del total de requerimientos de UCI que llegan a los hospitales públicos en Valparaíso, 94,33\%

Tabla 1. Número de traslados nacionales desde hospitales públicos a otros establecimientos

\begin{tabular}{|lrrrrrr|}
\hline Tipo de destino & $\mathbf{2 0 0 9}$ & $\mathbf{2 0 1 0}$ & $\mathbf{2 0 1 1}$ & $\mathbf{2 0 1 2}$ & $\mathbf{2 0 1 3}$ & Total \\
\hline Privado & 748 & 1.658 & 2.050 & 1.982 & 954 & 7.392 \\
\hline Púbico & 456 & 1.010 & 604 & 656 & 279 & 3.005 \\
\hline Total & 1.204 & 2.668 & 2.654 & 2.638 & 1.233 & 10.397 \\
\hline
\end{tabular}


Tabla 2. Número de traslados quinta región desde hospitales públicos a otros establecimientos

\begin{tabular}{|lrrrrrr|}
\hline Tipo de destino & $\mathbf{2 0 0 9}$ & $\mathbf{2 0 1 0}$ & $\mathbf{2 0 1 1}$ & $\mathbf{2 0 1 2}$ & $\mathbf{2 0 1 3}$ & Total \\
\hline Privado & 100 & 345 & 283 & 380 & 205 & 1.313 \\
\hline Público dentro de la región & 3 & 27 & 19 & 78 & 45 & 172 \\
\hline Público fuera de la región & 2 & 1 & 2 & 7 & 2 & 14 \\
\hline Total & 105 & 373 & 304 & 465 & 252 & 1.499 \\
\hline
\end{tabular}

fue atendido en el mismo establecimiento al que concurrieron al servicio de urgencia, $0,65 \%$ fue derivado a otro establecimiento público dentro de la región, $0,05 \%$ derivado a otro establecimiento público fuera de la región y $4,97 \%$ fue derivado a un establecimiento privado.

Es importante destacar que estas cifras tienen la limitante que son números en base a las atenciones en unidades de cuidados críticos que lograron ser trasladados a otros centros, pueden por lo tanto existir un número importante de pacientes que no logró recibir una atención especializada en unidades de cuidados críticos y que fallecieron en los distintos SUs, número que aún no ha sido determinado.

\section{Posibles soluciones a la saturación de los servicios de urgencia}

Reconociendo que el principal determinante del atochamiento del SU es el boarding, y que este está determinado por la carencia de disponibilidad rápida de camas en el resto del hospital, se han planteado múltiples estrategias, con resultados variables $^{17,23-25}$.

Un estudio, que buscaba evaluar los principales determinantes del desvío de ambulancias, a causa de la saturación de los servicios de urgencia, sacó a la luz el hecho de que el número de cirugías electivas programadas se asociaba estrechamente con el colapso de los SUs. El 2008, el Colegio Americano de Médicos de Urgencia (ACEP) sugirió como estrategia redistribuir las cirugías electivas hacia los días con menor flujo y suspender aquellas cirugías electivas para el mismo día, si la evaluación de necesidad de camas para descomprimir los SUs lo requiere ${ }^{17}$. Un estudio posterior, realizado en hospitales norteamericanos mostró, que hasta $11 \%$ y $14 \%$ de los hospitales habían adoptado estas medidas (redistribución de cirugías electivas y sus- pensión de cirugías electivas respectivamente $)^{26}$. Sin embargo, aún no hay estudios que analicen el real impacto de estas medidas.

En nuestro medio, esta estrategia también podría ser instaurada: un gran porcentaje de pacientes de neurocirugías o con cirugías abdominales y torácicas importantes electivas son trasladados a unidades de pacientes críticos para monitoreo estricto. Re-agendar estas cirugías para fines de semanas o días con menos requerimientos de camas de UCI, sin atrasarlas, podría ser una propuesta para mantener camas de cuidados críticos siempre disponibles para pacientes graves que ingresan por la urgencia.

Otra opción consiste en trasladar a los pacientes hospitalizados en el SU al piso que les corresponda lo antes posible, independientemente de la disponibilidad de camas. En muchos hospitales, los pacientes que son hospitalizados en servicios de urgencia ocupan un box de atención de forma prolongada, impidiendo la atención de otros pacientes en el mismo espacio físico. En algunos hospitales públicos, tanto de Estados Unidos de Norteamérica como en Chile, pacientes que ya han sido evaluados y están en espera de una cama en el hospital, son instalados en pasillos y áreas no médicas dentro del SU para habilitar estos boxes para la atención de pacientes nuevos. Así, en algunos hospitales se ha planteado y puesto en práctica una solución para estos pacientes, con el llamado "full capacity protocol" 24 . Cada vez que existe un grado de saturación importante en el $\mathrm{SU}$, aquellos pacientes que han sido hospitalizados ahí, ya sea en un pasillo o en un área no médica, y que cumplen ciertos criterios de estabilidad, son trasladados a los pasillos de los pisos correspondientes. Este modelo ha demostrado acortar de manera significativa los tiempos de espera por una cama definitiva y generar altas más precoces, esto sin afectar ni poner en riesgo a los pacientes sometidos a este protocolo ${ }^{27-28}$. Esto pudiera tener 
una explicación debido a que el personal médico y de enfermería del piso tiene mayor capacidad de acomodar las necesidades y evaluación de un paciente en el pasillo que la que tiene el personal del SU, quienes atienden a múltiples pacientes agudos a la vez. Como punto de apoyo, una encuesta hecha a pacientes sometidos y no sometidos a este protocolo mostró que los pacientes prefieren ser trasladados precozmente a un pasillo del piso correspondiente que a permanecer hospitalizados en el SU ${ }^{29}$. Aunque el full capacity protocol pareciera ser impracticable en el contexto de pacientes críticos, debe mencionarse que en muchas ocasiones la causa de la escasez de camas de UCI es la limitación de camas disponibles en servicios de menor complejidad para descarga. Una estrategia de full capacity desde la UCI o intermedio hacia pisos de cuidados básicos, en pacientes ya con orden de traslado, podría ayudar a descongestionar el acceso a camas de mayor complejidad desde la urgencia.

\section{Propuesta de alianza entre los servicios de urgencia y las unidades de cuidados intensivos como solución al problema de espera de camas críticas}

Como solución al problema de espera de camas críticas en situaciones de saturación y demora en el traslado de paciente a la unidad de cuidados críticos -que no involucran a las otras estrategias mencionadas anteriormente a nivel institucionalse han planteado protocolos de alianza o coordinación entre unidades de cuidados intensivos y el SU. Reconociendo que los servicios de urgencia se hacen cargo de un gran volumen de pacientes críticos, en Estados Unidos de Norteamérica (y probablemente a medida que la medicina de urgencia se establezca como especialidad médica en nuestro país) se ha propuesto la necesidad de formar sub-especialistas de urgencia en cuidados críticos $^{30}$.

También se han planteado estrategias de equipo, en donde un equipo de urgenciólogos y un equipo de enfermería son interconsultados al momento de tener un paciente hospitalizado en urgencia. Este equipo de urgenciólogos se hace cargo del paciente una vez que ha sido estabilizado, pero cuando aún se encuentra físicamente en el SU7.

Otro protocolo propuesto es el modelo cen- trado en la UCI (ICU centered model), donde el equipo de UCI se hace cargo del paciente desde el SU y el médico de urgencia está disponible sólo en las evaluaciones agudas. De esta manera, este equipo evaluaría y planificaría una estrategia de estudio y manejo, y el médico de urgencia sólo estaría atento en caso de descompensación ${ }^{7}$.

El último modelo que ha demostrado mejorar el desenlace y la continuidad del manejo de pacientes críticos en el servicio de urgencia está relacionado a la creación de protocolos de manejo en conjunto, entre los ambos equipos de profesionales $^{31}$.

\section{Conclusiones}

La limitada disponibilidad de camas críticas es una realidad con la que convive el sistema de salud de Chile, y constituye un importante problema de salud pública. La solución requerirá de tiempo y de una gran inversión. Esta carencia obliga a que muchas veces pacientes críticos se manejen por períodos prolongados en los SUs, lo que contribuye a la saturación y sobrepoblación de pacientes en estas áreas y además compromete su sobrevida, ya que el personal y el equipamiento están orientados a la estabilización a corto plazo.

Se pueden proponer soluciones orientadas a procesos, optimización de recursos y estructurales, al entender el sistema UCIs-SUs como un sistema integrado, con procesos y protocolos consensuados transversal y verticalmente. Asimismo, la integración de intensivistas en la toma de decisiones en los SU facilitará el ingreso de pacientes a áreas críticas y mejorará la gestión de los intensivos. Por otro lado, se beneficia la docencia e investigación en áreas de interés común y se enriquece la formación de los especialistas de ambas disciplinas.

\section{Referencias}

1. Nguyen HB, Rivers EP, Havstad S, Knoblich B, Ressler JA, Muzzin AM, et al. Critical care in the emergency department: A physiologic assessment and outcome evaluation Acad Emerg Med 2000; 7 (12): 1354-61.

2. Cardoso LT, Grion CM, Matsuo T, Anami EH, Kauss IA, Seko L, et al. Impact of delayed admission to intensive care units on mortality of critically ill patients: a 
cohort study. Crit Care 2011; 15 (1): R28. doi: 10.1186/ cc9975. Epub 2011.

3. Cline SD, Schertz RaK, Feucht EC. Expedited admission of patients decreases duration of mechanical ventilation and shortens ICU stay. Am J Emerg Med 2009; 27 (7): 843-6. doi:10.1016/j.ajem.2008.04.018.

4. Duke G, Green J, Briedis J. Survival of critically ill medical patients is time-critical. Crit Care Resusc 2004; 6 (4): 261-7.

5. Perkins J, Motov S. Clinical Practice Committee Statement: Mortality Related to Delay of ICU Patient Transfer from the Emergency. Available online: http:// www.aaem.org/UserFiles/file/icu_transfer_statement. pdf2011:14-15.

6. Varon J, Fromm RE, Levine RL. Emergency department procedures and length of stay for critically ill medical patients. Ann Emerg Med 1994; 23 (3): 546-9.

7. Cowan RM, Trzeciak S. Clinical review: Emergency department overcrowding and the potential impact on the critically ill. Crit Care 2005; 9 (3): 291-5. doi:10.1186/ cc2981.

8. Society of critical care medicine. Available at: http:// www.myicucare.org/Pages/default.aspx. Accessed September 29, 2014.

9. Hillman K. Critical care without walls. Curr Opin Crit Care 2002; 8 (6): 594-9.

10. Levy MM, Dellinger RP, Townsend SR, Linde-Zwirble WT, Marshall JC, Bion J, et al. Surviving Sepsis Campaign et al. The Surviving Sepsis Campaign: results of an international guideline-based performance improvement program targeting severe sepsis. Crit Care Med 2010; 38 (2): 367-74. doi:10.1097/CCM.0b013e$3181 \mathrm{cb} 0 \mathrm{cdc}$.

11. Khot UN, Johnson ML, Ramsey C, Khot MB, Todd R, Shaikh SR, et al. Emergency department physician activation of the catheterization laboratory and immediate transfer to an immediately available catheterization laboratory reduce door-to-balloon time in ST-elevation myocardial infarction. Circulation 2007; 116 (1): 67-76.

12. Tiel Groenestege-Kreb D, van Maarseveen O, Leenen L. Trauma team. Br J Anaesth 2014; 113 (2): 258-65.

13. Jauch EC, Saver JL, Adams HP Jr, Bruno A, Connors JJ, Demaerschalk BM, et al. Guidelines for the early management of patients with acute ischemic stroke: a guideline for healthcare professionals from the American Heart Association/American Stroke Association. Stroke 2013; 44 (3): 870-947.

14. Pines JM, Hilton JA, Weber EJ, Alkemade AJ, Al Shabanah $\mathrm{H}$, Anderson $\mathrm{PD}$, et al. International perspectives on emergency department crowding. Acad Emerg Med
2011; 18 (12): 1358-70.

15. Derlet Ro, Richards J, Kravitz R. Frequent Overcrowding in US Emergency departments. Acad Emerg Med 2001; 8 (2): 151-5.

16. Estadísticas de Atenciones de Urgencia-DEIS. Available at: http://www.deis.cl/?p=41. Accessed September 30, 2014.

17. Blum FC, Virginia W, Broida RI, Officer CO. Emergency Department Crowding: High-Impact Solutions. ACEP task force Reporty Board 2008; (April). Available at: www.acep.org/workarea/DownloadAsset. aspx?id=50026.

18. Gifford MJ, Franaszek JB, Gibson G. Emergency physicians' and patients' assessments: Urgency of need for medical care. Ann. Emerg. Med 1980; 9 (10): 502-7.

19. Hunt RC, De Hart KL, Allison EJ, Whitley TW. Patient and physician perception of need for emergency medical care: a prospective and retrospective analysis. Am J Emerg Med 1996; 14 (7): 635-9.

20. Emergency Department Crowding: High-Impact Solutions Continuing Medical Education Credit Information. American Collegue of Emergency Physicians Crowding: High-Impact Solutions. Avalilable at http://www.acep.org/workarea/DownloadAsset. aspx?id $=50026$

21. Singer AJ, Thode HC, Viccellio P, Pines JM. The association between length of emergency department boarding and mortality. Acad Emerg Med 2011; 18 (12): 1324-9.

22. Chalfin DB, Trzeciak S, Likourezos A, Baumann BM, Dellinger RP. Impact of delayed transfer of critically ill patients from the emergency department to the intensive care unit. Crit Care Med 2007; 35 (6): 1477-83.

23. Derlet RW, Richards JR. Ten solutions for emergency department crowding. West J Emerg Med 2008; 9 (1): 24-7.

24. Viccellio P. Emergency department overcrowding: an action plan. Acad Emerg Med 2001; 8 (2): 185-7.

25. Higginson I. Emergency department crowding. Emerg Med J 2012; 29 (6): 437-43.

26. Liu SW, Hamedani AG, Brown DFM, Asplin B, Camargo CA Jr. Established and novel initiatives to reduce crowding in emergency departments. West J Emerg Med 2013; 14 (2): 85-9.

27. Reducing Emergency Department Crowding Through the Full Capacity Protocol-Robert Wood Johnson Foundation. Available at: http://www.rwjf.org/en/about-rwjf/ newsroom/newsroom-content/2008/06/reducing-emergency-department-crowding-through-the-full-capacity. html. Accessed September 30, 2014.

28. Viccellio A, Santora C, Singer AJ, Thode HC, Henry 
MC. The association between transfer of emergency department boarders to inpatient hallways and mortality: a 4-year experience. Ann Emerg Med 2009; 54 (4): 487-91.

29. Viccellio P, Zito JA, Sayage V, Chohan J, Garra G, Santora $\mathrm{C}$, et al. Patients overwhelmingly prefer inpatient boarding to emergency department boarding. J Emerg Med 2013; 45 (6): 942-6.
30. Melanson P. Critical care medicine as a subspecialty of emergency medicine. CJEM 2000; 2 (4): 258-61.

31. Fogarty E, Saunders J, Cummins F. The effect of boarders on emergency department process flow. J Emerg Med 2014; 46 (5): 706-10.

32. Little JDC. A Proof for the Queuing Formula: $\lambda W$. Operations Research 1961; 9 (3): 383-7. 